\title{
PROSPECT, A Precision Reactor Oscillation and SPECTrum Short-Baseline Antineutrino Experiment
}

\author{
Xianyi Zhang* \\ On Behalf of PROSPECT Collaboration \\ Illinois Institute of Technology \\ E-mail: xzhan1350hawk.iit.edu
}

\begin{abstract}
PROSPECT, the Precision Reactor Oscillation and Spectrum, is a short-baseline reactor antineutrino experiment. PROSPECT consists of a segmented 4-ton ${ }^{6} \mathrm{Li}$ liquid scintillator antineutrino detector that will precisely measure the ${ }^{235} \mathrm{U}$ fission antineutrino spectrum from the High-Flux Isotope Reactor at Oak Ridge National Laboratory. PROSPECT's high statistics and high resolution measurements of the antineutrino energy spectrum and flux from HFIR's $235 \mathrm{U}$ core will be vital to understanding the discrepancies between predicted and measured antineutrino spectra and fluxes observed in previous commercial power reactor neutrino experiments; in addition, PROSPECT will search for the existence of sterile neutrino oscillations at the $\mathrm{eV}^{2}$-scale. PROSPECT's assembly was completed in late 2017 and physics data taking at HFIR began in 2018. This talk explains PROSPECT's physics objectives, describe its experimental design, and cover its installation and initial data-taking.
\end{abstract}

ICHEP 2018, International Conference on High Energy Physics

July 4-11, 2018

Seoul, South Korea

${ }^{*}$ Speaker. 


\section{Introduction}

Reactor antineutrino experiments shed light in the discovery of neutrino [1] and precise measurements of neutrino mixing angle $\theta_{13}[2,3,4]$. The short baseline flux measurements of $\bar{v}_{e}$ from commercial fission reactors found $\sim 6 \%$ discrepancy from the theoretical based prediction[5, 6], which often referred as Reactor Antineutrino Anomaly[7] (RAA). Those experiments revealed a $\bar{v}_{e}$ prompt energy spectrum distortion indicating 8-10\% excess at 4-6 MeV compared with the nuclear models. These results suggested reexaminations of fission branches that contributed to the antineutrino flux and spectrum anomaly. The flux deficit hinted a $\Delta m^{2} \sim \mathrm{eV}^{2}$ sterile neutrino oscillation[8]. The community has an urgent need of testing the possible sterile neutrino oscillation, as well as precise inputs of $\bar{v}_{e}$ flux and spectra from the major branches of fission reactors.

\section{Experiment Design}

PROSPECT, the Precision Reactor Oscillation and SPECTrum experiment, started taking data from the first quarter of 2018 to measure the baseline dependent $\bar{v}_{e}$ spectrum in 7-9 $\mathrm{m}$ from a Highly Enriched ${ }^{235} \mathrm{U}$ (HEU) reactor, the High Flux Isotopic Reactor (HFIR) at Oak Ridge National Laboratory (ORNL) (see, e.g., Figure 1). The goals of this experiment are to test the possible sterile neutrino oscillation and investigate the isotopic contribution to the spectrum distortion by measuring the absolute $\bar{v}_{e}$ spectrum from ${ }^{235} \mathrm{U}$.

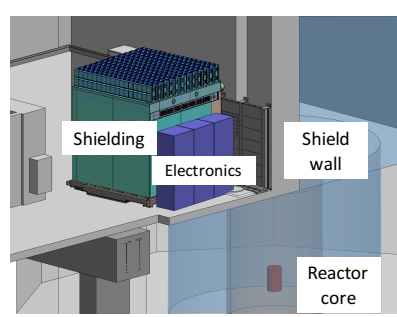

Figure 1: The layout of the PROSPECT experiment, where detector (green) is approximately $7-9 \mathrm{~m}$ from the reactor core (red)[9].

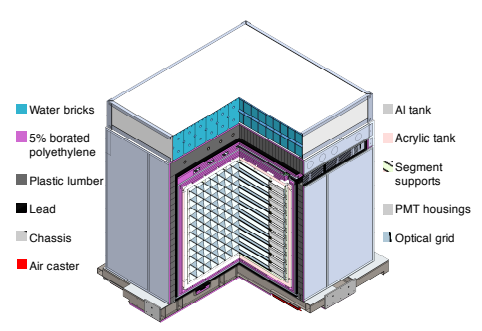

Figure 2: A view of the AD showing the shielding layers, the optical segmentation and PMTs with protective housings[9].

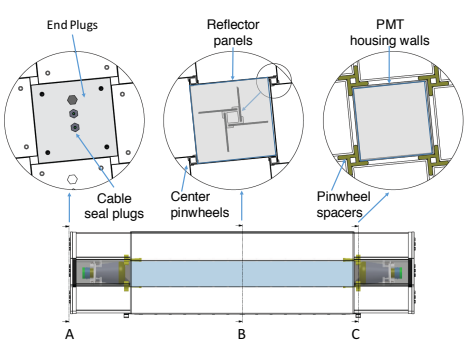

Figure 3: The individual segment of AD. (Top left) The end of the PMT housing; (Top middle) The cross section of the segment; (Top right) The front face of PMT housing.

The HFIR is an 85 MW compact cylindrical core, from which $>99 \%$ of $\bar{v}_{e}$ are generated from ${ }^{235} \mathrm{U}$, with reactor cycle of $\sim 24$ days per cycle. The PROSPECT Antineutrino Detector (AD) is an optically segmented Liquid Scintillator (LS), which contains $\sim 4$ tons of ${ }^{6} \mathrm{Li}$ loaded EJ-309 (see, e.g., Figure 2). To precisely measure event energy and position, the LS was uniformly divided into $14 \times 11$ meter-long longitudinal segments. Each segment was enclosed by 4 reflective and low mass separators and two 5" Photomultiplier Tubes (PMTs) with one PMT on each end. The scintillation light is constrained in a small number of segments, and the vertex position along each segment is reconstructed based on differences of timing and light integral between the two PMTs. The separators were interlocked by the hollow supporting rods showed in Figure 3, which allows 
the calibration sources to be inserted into the detector. The shielding of PROSPECT AD consists of water bricks on top, polyethylene, lead and borated polyethylene containing the detector in layers, to minimize the reactor correlated and cosmogenic backgrounds.

The PROSPECT AD detects Inverse Beta Decay (IBD), $\bar{v}_{e}+p \rightarrow e^{+}+n$. The $e^{+}$signal is defined as the prompt signal, whose energy is dependent on the incident $\bar{v}_{e}$ energy. The IBD neutrons are mostly captured by ${ }^{6} \mathrm{Li} 40-50 \mu$ s after the prompt signal. The delayed n-Li signal, which emits an $\alpha$ particle and a ${ }^{3} \mathrm{~T}$, can be tagged by pulse shape discrimination. Besides, we enhanced the signal to background ratio with the event selections: 1) cosmic ray and fast neutron veto time; 2) prompt-delay timing and topological proximation; 3 ) fiducial volume of detector that excludes the outermost layer of segments.

\section{Detector Calibration}

We inserted optical LED pulses, radioactive sources and used the ambient data to calibrate the detector's response. The $795 \pm 15 \mathrm{PE} / \mathrm{MeV}$ photon statistics of the LS, which dominates the energy resolution, was characterized by the optical calibration. With the ${ }^{137} \mathrm{Cs}$ source deployed throughout the detector, we found the relative energy scale uniformity within $\sim 1 \%$ among detector segments. The energy scale (energy resolution) varied within $\sim 1 \%(\sim 10 \%)$ during the unblinded data period. The resolution of position reconstruction along the segment axial direction was $\sim 5 \mathrm{~cm}[10]$.

\section{Searching for Sterile Neutrino}

From 0.8 to $7.2 \mathrm{MeV}$ of reconstructed prompt energy, we collected 56378 IBD candidates during 33 reactor-on days and 28 reactor-off days. The amount of IBD events after correlated and accidental background subtraction was $25461 \pm 283$, indicating 2.20 (1.32) correlated (accidental) signal to background ratio.

To test the sterile neutrino oscillation independently from nuclear models, the fiducial AD segments were divided into 6 groups with respect to their baselines. The spectrum of prompt IBD energy was measured by the fiducial detector and individually by each of the segment groups. The comparison between the spectrum from each group and the summed spectrum is shown in Figure 4. The $\chi^{2}$ test between the baseline dependent spectra and the full-detector-measured absolute spectrum was evaluated through a combined covariance matrix of statistical and systematic uncertainties. The exclusion curve showed in Figure 5, which was based on Feldman-Cousins[11] confidence interval. As a result, we disfavored the RAA best fit model at $2.2 \sigma$.

\section{Conclusions}

PROSPECT is a reactor antineutrino experiment built to test the possible $\Delta m^{2} \sim \mathrm{eV}^{2}$ sterile neutrino oscillation and precisely measure $\bar{v}_{e}$ spectrum from ${ }^{235} \mathrm{U}$. We characterized the stability of event and uncertainties of the energy and position reconstruction at an early stage. The PROSPECT $\mathrm{AD}$ observed reactor antineutrinos in the first $2 \mathrm{hr}$ of data and collected $25461 \pm 283$ reactor correlated $\bar{v}_{e}$ events in 33 reactor-on days. The model-independent oscillation analysis disfavored the RAA's for best fit sterile neutrino oscillation with $2.2 \sigma$ confidence level. 


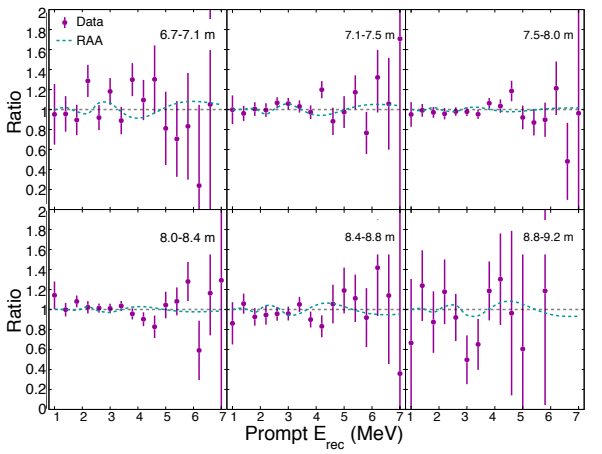

Figure 4: The ratio of the IBD spectra measured in six baselines to the summed energy spectra, where the purple dashed line indicates nooscillation scenario and green dashed line highlights RAA best fit model [10].

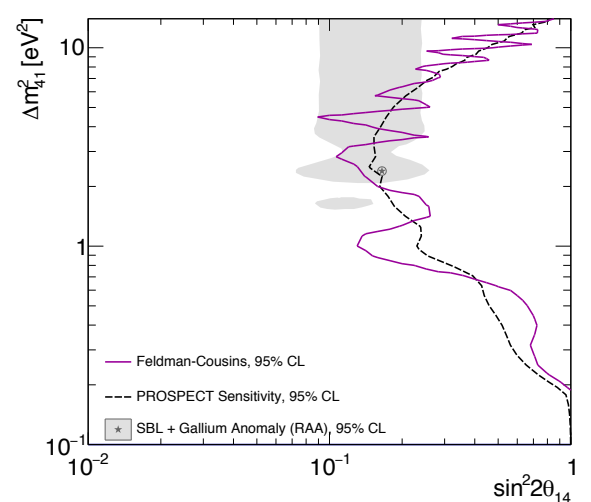

Figure 5: The sensitivity to sterile neutrino oscillation and exclusion curve with $95 \%$ confidence level, disfavoring the RAA best fit at $2.2 \sigma[10]$.

\section{References}

[1] C. L. Cowan, F. Reines, F. B. Harrison, H. W. Kruse and A. D. McGuire, Detection of the free neutrino: A Confirmation, Science 124 (1956) 103.

[2] Daya Bay Collaboration collaboration, F. P. An, J. Z. Bai, A. B. Balantekin, H. R. Band, D. Beavis, W. Beriguete et al., Observation of electron-antineutrino disappearance at daya bay, Phys. Rev. Lett. 108 (2012) 171803.

[3] RENO Collaboration collaboration, J. K. Ahn, S. Chebotaryov, J. H. Choi, S. Choi, W. Choi, Y. Choi et al., Observation of reactor electron antineutrinos disappearance in the reno experiment, Phys. Rev. Lett. 108 (2012) 191802.

[4] Double Chooz Collaboration collaboration, Y. Abe, C. Aberle, T. Akiri, J. C. dos Anjos, F. Ardellier, A. F. Barbosa et al., Indication of reactor $\bar{v}_{e}$ disappearance in the double chooz experiment, Phys. Rev. Lett. 108 (2012) 131801.

[5] P. Huber, Determination of antineutrino spectra from nuclear reactors, Phys. Rev. C 84 (2011) 024617.

[6] T. A. Mueller, D. Lhuillier, M. Fallot, A. Letourneau, S. Cormon, M. Fechner et al., Improved predictions of reactor antineutrino spectra, Phys. Rev. C 83 (2011) 054615.

[7] G. Mention, M. Fechner, T. Lasserre, T. A. Mueller, D. Lhuillier, M. Cribier et al., Reactor antineutrino anomaly, Phys. Rev. D 83 (2011) 073006.

[8] K. N. Abazajian et al., Light Sterile Neutrinos: A White Paper, 1204.5379.

[9] PROSPECT collaboration, J. Ashenfelter et al., The PROSPECT Reactor Antineutrino Experiment, 1808.00097.

[10] PROSPECT collaboration, J. Ashenfelter et al., First search for short-baseline neutrino oscillations at HFIR with PROSPECT, 1806.02784.

[11] G. J. Feldman and R. D. Cousins, Unified approach to the classical statistical analysis of small signals, Phys. Rev. D 57 (1998) 3873. 